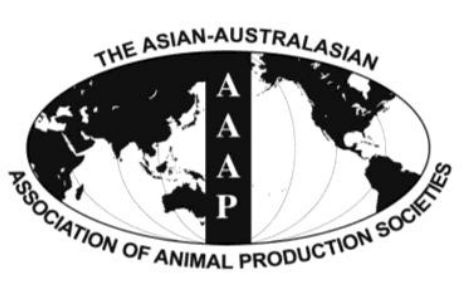

Open Access

Asian Australas. J. Anim. Sci.

Vol. 27, No. 3 : 357-364 March 2014

http://dx.doi.org/10.5713/ajas.2013.13405

www.ajas.info

pISSN 1011-2367 elSSN 1976-5517

\title{
Nutrient Digestibility, Ruminal Fermentation Activities, Serum Parameters and Milk Production and Composition of Lactating Goats Fed Diets Containing Rice Straw Treated with Pleurotus ostreatus
}

\author{
A. E. Kholif ${ }^{1}$, H. M. Khattab ${ }^{2}$, A. A. El-Shewy ${ }^{1}$, A. Z. M. Salem ${ }^{3,4, *}$, \\ A. M. Kholif ${ }^{1}$, M. M. El-Sayed ${ }^{1}$, H. M. Gado ${ }^{2}$, and M. D. Mariezcurrena ${ }^{5}$ \\ ${ }^{1}$ Dairy Science Department, National Research Centre, El Buhoth Street, Dokki, Cairo, Egypt
}

\begin{abstract}
The study evaluated replacement of Egyptian berseem clover (BC, Trifolium alexandrinum) with spent rice straw (SRS) of Pleurotus ostreatus basidiomycete in diets of lactating Baladi goats. Nine lactating homo-parity Baladi goats (average BW $23.8 \pm 0.4$ $\mathrm{kg}$ ) at $7 \mathrm{~d}$ postpartum were used in a triplicate $3 \times 3$ Latin square design with $30 \mathrm{~d}$ experimental periods. Goats were fed a basal diet containing 0 (Control), 0.25 (SRS25) and 0.45 (SRS45) (w/w, DM basis) of SRS. The Control diet was berseem clover and concentrate mixture (1:1 DM basis). The SRS45 had lowered total feed intake and forages intake compared to Control. The SRS25 and SRS45 rations had the highest digestibilities of DM $(p=0.0241)$ and hemicellulose $(p=0.0021)$ compared to Control which had higher $(\mathrm{p}<0.01)$ digestibilities of OM $(\mathrm{p}=0.0002)$ and CP $(\mathrm{p}=0.0005)$ than SRS25 and SRS45. Ruminal $\mathrm{pH}$ and microbial protein synthesis were higher $(\mathrm{p}<0.0001)$ for SRS25 and SRS45 than Control, which also had the highest $(\mathrm{p}<0.0001)$ concentration of TVFA, total proteins, non-protein $\mathrm{N}$, and ammonia-N. All values of serum constituents were within normal ranges. The Control ration had higher serum globulin $(p=0.0148)$, creatinine $(p=0.0150)$, glucose $(p=0.0002)$ and cholesterol $(p=0.0016)$. Both Control and SRS25 groups had the highest $(p<0.05)$ milk $(p=0.0330)$ and energy corrected milk $(p=0.0290)$ yields. Fat content was higher $(p=0.0373)$ with SRS45 and SRS25 groups compared with Control. Replacement of BC with SRS in goat rations increased milk levels of conjugated linoleic acid and unsaturated fatty acids compared with Control. It was concluded that replacing 50\% of Egyptian berseem clover with SRS in goat rations improved their productive performance without marked effects on metabolic indicators health. (Key Words: Fungus, Goats, Milk, Spent Rice Straw)
\end{abstract}

\section{INTRODUCTION}

Agricultural policy in Egypt is aimed to increase the

\footnotetext{
* Corresponding Author: A. Z. M. Salem. Tel: +20-521-722-296-55-

42, Fax: +20-521-722-180-61-94, E-mail: asalem70@yahoo.com

${ }^{2}$ Animal Production Department, Faculty of Agriculture, Ain Shams University, Cairo, Egypt.

${ }^{3}$ Facultad de Medicina Veterinaria y Zootecnia, Universidad Autónoma del Estado de México, Estado de México, México.

${ }^{4}$ Faculty of Agriculture, Alexandria University, Alexandria, Egypt.

${ }^{5}$ Facultad de Ciencias Agrícolas, Universidad Autónoma del Estado de México, Estado de México, México.

Submitted Jul. 8, 2013; Accepted Sept. 26, 2013; Revised Nov. 4, 2013
}

area cultivated by alternative crops such as berseem clover (BC). Rice straw (RS) in Egypt, is a potential feed during the fall and winter for many small-scale livestock owners when rotational BC is unavailable. However only $\sim 25 \%$ of the total amount of RS is fed to livestock (Steele et al., 2009). Use of RS as an animal feed is limited by its low nutritive value, mainly attributable to the crystalline structure of the cellulose fibrils surrounded by hemicellulose, and by the presence of lignin, which prevents enzymes penetration (Chahal and Chahal, 1998).

Biological delignification of straws by white-rot fungi may be a promising way to improve their nutritive value (Fazaeli et al., 2002). The organisms predominantly responsible for lignocellulose degradation are fungi, and the

Copyright $@ 2014$ by Asian-Australasian Journal of Animal Sciences This is an open-access article distributed under the terms of the Creative Commons Attribution Non-Commercial License (http://creativecommons.org/licenses/by-nc/3.0/), which permits unrestricted non-commercial use, distribution, and reproduction in any medium, provided the original work is properly cited. 
most effective are basidiomycetes (Rabinovich et al., 2004). Fungal lignocellulolytic enzymes break the polysaccharidelignin complex. This would enhance the accessibility of enzymes to potentially digestible biomass resulting in higher degradation of the straw, which may create a more nutritious feed (Tawffek, 2011). Pleurotus fungi can grow on straw and decompose its structural carbohydrate (Fazaeli et al., 2002). The potential of Pleurotus ostreatus to reduce indigestible cell wall components and increase cell wall digestibility of straw has been reported (Fazaeli et al., 2002).

The aim of our study was to evaluate utilization of spent RS (SRS), by products of Pleurotus ostreatus cultivation, in the diet of dairy goats, and to assess the productive performance of lactating Baladi goats fed different levels of SRS as a replacement for BC.

\section{MATERIALS AND METHODS}

\section{Spent rice straw preparation}

Mushroom cultivation was completed out at a mushroom farm at Cairo (Egypt) according to Oei (2005).

Briefly, The RS was weighed and soaked in water overnight and air-dried for $15 \mathrm{~min}$. About $5 \mathrm{~kg}$ calcium carbonate/100 kg RS (w/w; DM basis) was added to maintain $\mathrm{pH}$ neutrality. The prepared $\mathrm{RS}$ was sterilized with $100^{\circ} \mathrm{C}$ water for $1 \mathrm{~h}$ and then after cooling to $20^{\circ} \mathrm{C}$ to $25^{\circ} \mathrm{C}$, they were put into plastic bags $(40 \times 70 \mathrm{~cm}$ high). Substrates were inoculated with the fungus $P$. ostreatus. Bags were tightly closed, and pin holes were made on the surfaces, after which they were kept in at $25^{\circ} \mathrm{C}$ in the dark until mycelium formed for $\sim 21 \mathrm{~d}$. After mycelium formation, large holes were made in the polythene bags to allow normal development of fruit bodies. After mycelium formation, bags were put in growing rooms $22^{\circ} \mathrm{C}$ with a 12 h photoperiod (at 1,500 to 2,000 Lux) and $85 \%$ to $90 \%$ relative humidity. Adequate ventilation was provided to prevent increases of carbon dioxide concentration. Bags were collected after 7 weeks of incubation when the mushroom fruits were harvested twice, and sun dried.

\section{Animals, feeding and experimental design}

Nine Baladi goats of $23.8 \pm 0.4 \mathrm{~kg}$ of $\mathrm{BW}$ were divided into 3 groups of 3 goats. The goats were housed in tie stalls with free access to water. Three diets were formulated with 50:50 (DM) forage:concentrates ratio to meet their nutrient requirements when fed at $3 \%$ of BW (which was changed continuously based on changes in BW; Khattab et al., 2011). The Control diet consisted of Egyptian berseem clover (Trifolium alexandrinum) and concentrate mixture on a 1:1 DM basis. The other two diets contained 0.25 (SRS25) and 0.45 (SRS45) (w/w, DM basis) of SRS. Diets were fed to each goat at 08:00 and 16:00 $\mathrm{h}$ in equal portions. The ingredient and nutrient composition of the diets are in Table 1 .

The experimental periods were $30 \mathrm{~d}$ in a triplicate $3 \times 3$ Latin square design (three animals per cell). Feed intake and milk yield were measured on the last $7 \mathrm{~d}$ of each experimental period. Goats were hand milked at 09:00 and 21:00 $\mathrm{h}$, and portions according to milk yield at each milking pooled for daily milk samples.

Fecal grab samples were collected daily during the last $3 \mathrm{~d}$ of each experimental period, dried at $60^{\circ} \mathrm{C}$ in a forcedair oven for $48 \mathrm{~h}$ and pooled by goat within period. Acid insoluble ash was used as an internal indigestibility marker where coefficients of digestion calculated according to Ferret et al. (1999).

\section{Ruminal fermentation and blood chemistry}

On the last day of each experimental period,

Table 1. Chemical composition of dietary ingredients and experimental rations, $\mathrm{g} / \mathrm{kg}$ on DM basis

\begin{tabular}{|c|c|c|c|c|c|c|c|c|c|c|c|c|c|}
\hline & $\begin{array}{c}\text { Dry } \\
\text { matter }\end{array}$ & $\begin{array}{l}\text { Organic } \\
\text { matter }\end{array}$ & $\begin{array}{l}\text { Crude } \\
\text { protein }\end{array}$ & $\begin{array}{c}\text { Ether } \\
\text { extract }\end{array}$ & $\begin{array}{l}\text { Neutral } \\
\text { detergent } \\
\text { fiber }\end{array}$ & $\begin{array}{c}\text { Acid } \\
\text { detergent } \\
\text { fiber }\end{array}$ & $\begin{array}{c}\text { Acid } \\
\text { detergent } \\
\text { lignin }\end{array}$ & Hemicellulose & Cellulose & $\begin{array}{l}\text { Soluble } \\
\text { protein }\end{array}$ & $\begin{array}{c}\text { Insoluble } \\
\text { protein }\end{array}$ & $\begin{array}{c}\text { True } \\
\text { protein } \mathrm{N}\end{array}$ & $\begin{array}{c}\text { Non- } \\
\text { protein } \\
\mathrm{N}\end{array}$ \\
\hline \multicolumn{14}{|c|}{ Dietary ingredients } \\
\hline $\begin{array}{c}\text { Berseem } \\
\text { clover }\end{array}$ & 104.0 & 851.6 & 139.2 & 38.0 & 506.3 & 349.3 & 49.6 & 156.9 & 299.8 & 674.0 & 326.0 & 717.9 & 282.1 \\
\hline $\begin{array}{l}\text { Spent rice } \\
\text { straw }\end{array}$ & 830.9 & 708.9 & 116.5 & 26.5 & 396.1 & 301.9 & 42.8 & 94.2 & 259.1 & 547.0 & 453.0 & 813.3 & 186.7 \\
\hline $\begin{array}{c}\text { Concentrate } \\
\text { mixture }^{1}\end{array}$ & 863.2 & 863.8 & 170.9 & 60.0 & 455.9 & 257.1 & 47.8 & 198.8 & 209.3 & - & - & - & - \\
\hline \multicolumn{14}{|c|}{ Experimental rations ${ }^{2}$} \\
\hline Control & 483.6 & 857.7 & 155.0 & 49.0 & 481.1 & 303.2 & 48.7 & 177.9 & 254.5 & - & - & - & - \\
\hline SRS25 & 665.3 & 822.0 & 149.4 & 46.1 & 453.6 & 291.4 & 47.0 & 162.2 & 244.4 & - & - & - & - \\
\hline SRS45 & 810.7 & 793.5 & 144.8 & 43.8 & 431.5 & 281.9 & 45.6 & 149.7 & 236.2 & - & - & - & - \\
\hline
\end{tabular}

${ }^{1}$ Concentrate mixture: $25 \%$ un-decorticated cotton seed cake, $21 \%$ wheat bran, $50 \%$ yellow corn, $2 \%$ limestone and $1 \%$ salt, $1 \%$ mineral and vitamin mixture (contained, per kg: $4.5 \% \mathrm{Ca}, 2.5 \% \mathrm{P}, 6.6 \% \mathrm{Na}, 1.5 \% \mathrm{Mg}, 1.2 \% \mathrm{~K}, 0.11 \% \mathrm{~S}, 1,372 \mathrm{mg}$ of Fe, $1,032 \mathrm{mg}$ of $\mathrm{Mn}, 1,500 \mathrm{mg}$ of $\mathrm{Zn}, 247 \mathrm{mg}$ of $\mathrm{Cu}$, $16 \mathrm{mg}$ of I, $16 \mathrm{mg}$ of Co, $10 \mathrm{mg}$ of Se, 185,000 IU of vitamin A, 32,500 IU of vitamin D3, and $900 \mathrm{IU}$ of vitamin E).

${ }^{2}$ Control $=50 \%$ concentrate mixture $+50 \%$ berseem clover, $\mathrm{T} 1=50 \%$ concentrate mixture $+25 \%$ berseem clover $+25 \%$ spent rice straw, $\mathrm{T} 2=50 \%$ concentrate mixture $+5 \%$ berseem clover $+45 \%$ spent rice straw.

${ }^{3}$ Calculated according to NRC (2001). 
fermentation characteristics were determined at 0,3 , and 6 $\mathrm{h}$ post morning feeding. Rumen contents $(100 \mathrm{~mL})$ were sampled in the area of the ventral blind sac with a composite sample strained through 4 layers of cheesecloth. The $\mathrm{pH}$ of ruminal fluid was immediately determined using a pH meter (model M90, Corning Inc., Corning, NY, USA). Strained rumen liquor was stored in $45 \mathrm{~mL}$ glass bottles, with adding few drops of toluene and paraffin oil just to cover the surface, and stored at $-20^{\circ} \mathrm{C}$ for later analysis of total $\mathrm{N}(\mathrm{TN})$, non-protein $\mathrm{N}(\mathrm{NPN})$, ammonia-N, microbial protein and total volatile fatty acid (TVFA).

On the last day of the experimental period, $10 \mathrm{~mL}$ of blood from each goat was collected into a clean dry tube from the jugular vein $4 \mathrm{~h}$ after feeding. Blood samples were centrifuged at $4,000 \times g$ at $4^{\circ} \mathrm{C}$ for $20 \mathrm{~min}$. Serum was separated into clean dried glass vials and frozen at $-20^{\circ} \mathrm{C}$ until analysis.

\section{Chemical analysis}

Dried feed samples was ground through a Wiley mill (Arthur H. Thomas, Philadelphia, PA, USA) using a $1 \mathrm{~mm}$ screen. Samples of feed, feed orts and feces were analyzed for DM (\#930.15), N (\#954.01), and ether extract (EE; \#920.39), according to AOAC (1995), while fiber fractionation (i.e. neutral detergent fiber, NDF and acid detergent fiber, ADF) were completed according to Van Soest et al. (1991). Protein solubility of some ingredients were determined according to Araba and Dale (1990). True protein was determined according to Kim et al. (2011) using trichloroacetic acid. NPN was calculated by deducting true protein $\mathrm{N}$ from TN. Milk samples were analyzed using Foss 120 Milko-Scan, Foss Electric, Hillerød, Denmark.

A digital $\mathrm{pH}$ meter with a glass electrode was used to determine ruminal $\mathrm{pH}$, which were also analyzed for TVFA according to Warner (1964). TN, NPN and ammonia N were analyzed as described by AOAC (1995). Microbial N was determined according to the method of Makkar et al. (1982) using tungstic acid.

Serum samples were analyzed using specific kits obtained from Stanbio Laboratory, Boerne, Texas, USA. Total protein (Cannon et al., 1974), albumin (Dumas and Biggs, 1972), urea (Henry et al., 1974), creatinine (Spierto et al., 1979), glucose (Howanitz and Howanitz, 1984), cholesterol (Stein, 1986) and triglycerides (Scheletter and Nussel, 1975). However, serum aspartate aminotransferase (AST) and alanine aminotransferase (ALT) were determined colorimetrically according to AST and ALT kits (Quimica Clinica Aplicada S.A., Spain) based on reaction of Reitman and Frankel (1957).

Milk fatty acids were determined using methyl esters prepared by base-catalyzed methanolysis of the glycerides ( $\mathrm{KOH}$ in methanol) according to International Standards (ISO-IDF, 2002). Fatty acid methyl esters were separated using a Cp-Sil 88 fused-silica capillary column (100 m× $0.25 \mathrm{~mm}$ i.d.x $0.2 \mu \mathrm{m}$ film thickness, Chrompack, Middelburg, Netherlands) on a Perkin-Elmer chromatograph (model 8420, Beaconsfield, Perkin Elmer, Beaconsfield, UK) equipped with a flame ionization detector.

\section{Calculations and statistical analysis}

Energy corrected milk was calculated according to Sjaunja et al. (1991).

All data except ruminal parameters were analyzed as a triplicate $3 \times 3$ Latin square using PROC MIXED of SAS (2001, Ver.8.02, SAS Institute Inc., Cary, NC, USA). The statistical model included a random effect of goat with period and treatment as fixed effects. The TVFA and ammonia $\mathrm{N}$ data were analyzed as repeated measurements in time with PROC MIXED of SAS using the following model:

$$
\mathrm{Y}_{\mathrm{ijkl}}=\mu+\mathrm{T}_{\mathrm{i}}+\mathrm{A}_{\mathrm{j}}\left(\mathrm{T}_{\mathrm{i}}\right)+\mathrm{S}_{\mathrm{k}}+(\mathrm{T} \times \mathrm{S})_{\mathrm{ik}}+\mathrm{E}_{\mathrm{ijkl}}
$$

where: Y expressed the every observation of the jth animal in the kth sampling time given ith treatment, $\mathrm{T}$ (1-3) expressed the treatments effect, A (T) expressed the animal within treatments, $S$ (1-3) expressed the sampling time effect, $\mathrm{T} \times \mathrm{S}$ expressed the interaction between the treatments and sampling times and $\mathrm{E}$ expressed the experimental error.

Differences among means were determined using Duncan's multiple range test (Duncan, 1955).

\section{RESULTS}

The energy values for the compounded rations, expressed as digestible energy (DE), metabolizable energy (ME) and net energy for lactation $\left(\mathrm{NE}_{\mathrm{L}}\right)$, declined slightly as SRS inclusion levels increased.

The SRS45 lowered $(\mathrm{p}=0.0114)$ total DM intake as well as DM intake from forages $(\mathrm{p}=0.0061)$ compared with Control (Table 2). However goats fed increasing levels of SRS consumed less $(\mathrm{p}=0.0061)$ DM from forage. Feeding SRS25 and SRS45 rations to goats increased $(\mathrm{p}<0.05)$ digestibilities of DM $(\mathrm{p}=0.0241)$ and hemicellulose ( $\mathrm{p}=0.0021)$ compared to Control. However the Control ration had higher digestibilities for OM ( $\mathrm{p}=$ $0.0002)$ and CP $(p=0.0005)$ than the SRS groups.

The Control ration had the highest $(\mathrm{p}<0.0001)$ concentrations of ruminal TVFA, TN, NPN, and ammonia$\mathrm{N}$ (Table 3). In contrast, the SRS25 and SRS45 groups had higher $(\mathrm{p}<0.0001)$ ruminal $\mathrm{pH}$ and microbial protein.

All serum constituents' value were within normal ranges (Table 4; Boyd, 2011). The Control ration increased ( $\mathrm{p}<$ $0.01)$ serum globulin $(\mathrm{p}=0.0148)$, creatinine $(\mathrm{p}=0.0150)$ and glucose $(\mathrm{p}=0.0002)$ compared to SRS25 and SRS45. 
Table 2. Effect of replacing berseem clover with spent rice straw on dry matter intake, nutrient digestibilities, and diets' nutritive values $(n=9)$

\begin{tabular}{|c|c|c|c|c|c|}
\hline & \multicolumn{3}{|c|}{ Diets $^{1}$} & \multirow{2}{*}{ SEM } & \multirow{2}{*}{$\mathrm{p}$ value } \\
\hline & Control & SRS25 & SRS45 & & \\
\hline \multicolumn{6}{|l|}{ Dry matter intake $(\mathrm{g} / \mathrm{d})$} \\
\hline Concentrate & 353.3 & 359.5 & 359.4 & 9.11 & 0.1400 \\
\hline Forage & $353.3^{\mathrm{a}}$ & $346.6^{\mathrm{ab}}$ & $326.7^{\mathrm{b}}$ & 9.20 & 0.0061 \\
\hline Total & 706.6 & 706.1 & 686.1 & 12.92 & 0.0114 \\
\hline \multicolumn{6}{|l|}{ Nutrient digestibilities (\%) } \\
\hline Dry matter & $57.88^{\mathrm{b}}$ & $62.36^{\mathrm{a}}$ & $59.18^{\mathrm{ab}}$ & 1.11 & 0.0241 \\
\hline Organic matter & $64.02^{\mathrm{a}}$ & $61.30^{\mathrm{a}}$ & $55.93^{\mathrm{b}}$ & 1.15 & 0.0002 \\
\hline Crude protein & $63.02^{\mathrm{a}}$ & $59.70^{\mathrm{ab}}$ & $56.01^{\mathrm{b}}$ & 1.09 & 0.0005 \\
\hline Ether extract & 59.71 & 60.42 & 61.73 & 1.19 & 0.5380 \\
\hline Neutral detergent fiber & $56.92^{\mathrm{b}}$ & $60.33^{\mathrm{a}}$ & $59.65^{\mathrm{ab}}$ & 1.10 & 0.0869 \\
\hline Acid detergent fiber & 59.80 & 60.80 & 60.86 & 1.12 & 0.7609 \\
\hline Hemicellulose & $52.45^{\mathrm{b}}$ & $59.52^{\mathrm{a}}$ & $57.52^{\mathrm{ab}}$ & 1.19 & 0.0021 \\
\hline Cellulose & 60.40 & 60.90 & 58.15 & 1.13 & 0.2145 \\
\hline $\mathrm{DCP}(\mathrm{g} / \mathrm{kg})^{2}$ & 97.7 & 89.2 & 81.1 & & \\
\hline $\mathrm{DE}(\mathrm{Mcal} / \mathrm{kg})^{2}$ & 2.5 & 2.4 & 2.2 & & \\
\hline $\operatorname{ME}(\mathrm{Mcal} / \mathrm{kg})^{2}$ & 2.1 & 1.9 & 1.8 & & \\
\hline $\mathrm{NE}_{\mathrm{L}}(\mathrm{Mcal} / \mathrm{kg})^{2}$ & 1.3 & 1.2 & 1.1 & & \\
\hline
\end{tabular}

While the SRS25 and SRS45 groups had lower $(\mathrm{p}<0.05)$ serum cholesterol $(\mathrm{p}=0.0016)$. There were no differences ( $>0.05)$ for serum total proteins, urea, AST, ALT, and triglycerides.

The SRS45 goats had lower $(\mathrm{p}=0.0330)$ milk and energy corrected milk ( $\mathrm{p}=0.0290)$ yields than Control and SRS25 (Table 5), but the SRS25 and SRS45 diets had higher $(\mathrm{p}=0.0373)$ milk fat than Control. They also had higher $(\mathrm{p}=0.0392)$ milk protein than Control.

The most abundant fatty acid (Table 6) for all treatments was palmitic acid $\left(\mathrm{C}_{16: 0}\right)$ followed by elaidic acid $\left(\mathrm{C}_{18: \ln 9 t}\right)$, capric acid $\left(\mathrm{C}_{10: 0}\right)$ and stearic acid $\left(\mathrm{C}_{18: 0}\right)$. The Control ration had higher values of saturated fatty acids $(p=$
$0.0461)$, medium-chain fatty acids ( $\mathrm{p}=0.4424)$, while the SRS25 and SRS45 rations had higher content of unsaturated fatty acids $(\mathrm{p}=0.0752)$, monounsaturated fatty acids $(\mathrm{p}=$ $0.7450)$, polyunsaturated fatty acids $(\mathrm{p}=0.0896)$, longchain fatty acids $(p=0.7065)$ and conjugated linoleic acid $(p=0.0445)$ compared to Control.

\section{DISCUSSION}

\section{Dry matter intake and nutrients digestibility}

The decreased forage DM with increasing levels of SRS in the diet may be because BC is more palatable than SRS, or as a result of excessive rumen undegradable protein

Table 3. Effect of replacing berseem clover with spent rice straw on ruminal fermentation parameters and microbial protein synthesis $(n=9)$

\begin{tabular}{|c|c|c|c|c|c|}
\hline & \multicolumn{3}{|c|}{ Diets $^{1}$} & \multirow{2}{*}{ SEM } & \multirow{2}{*}{$\mathrm{p}$ value } \\
\hline & Control & SRS25 & SRS45 & & \\
\hline $\mathrm{pH}$ & $6.49^{\mathrm{b}}$ & $6.63^{\mathrm{a}}$ & $6.64^{\mathrm{a}}$ & 0.092 & $<0.0001$ \\
\hline TVFA (meq/dL) & $10.8^{\mathrm{a}}$ & $9.3^{\mathrm{b}}$ & $8.9^{c}$ & 0.14 & $<0.0001$ \\
\hline Ammonia-N (mg/dL) & $23.8^{\mathrm{a}}$ & $20.6^{\mathrm{b}}$ & $19.0^{\mathrm{c}}$ & 3.23 & $<0.0001$ \\
\hline Microbial protein (mg/dL) & $146.5^{c}$ & $178.8^{\mathrm{a}}$ & $159.9^{b}$ & 5.22 & $<0.0001$ \\
\hline Total nitrogen (mg/dL) & $99.2^{\mathrm{a}}$ & $93.9^{b}$ & $90.1^{\mathrm{c}}$ & 3.33 & $<0.0001$ \\
\hline Non-protein nitrogen (mg/dL) & $42.0^{\mathrm{a}}$ & $35.8^{\mathrm{b}}$ & $32.5^{\mathrm{c}}$ & 3.50 & $<0.0001$ \\
\hline True protein nitrogen $(\mathrm{mg} / \mathrm{dL})$ & $57.2^{b}$ & $58.1^{\mathrm{a}}$ & $57.7^{b}$ & 3.40 & $<0.0001$ \\
\hline
\end{tabular}

Means with different superscripts in the same row differ $(\mathrm{p}<0.05)$.

${ }^{1}$ Diets contained per kg: Control $=500 \mathrm{~g}$ concentrate mixture $+500 \mathrm{~g}$ berseem clover, $\mathrm{SRS} 25=500 \mathrm{~g}$ concentrate mixture $+250 \mathrm{~g}$ berseem clover $+250 \mathrm{~g}$ spent rice straw; SRS $45=500 \mathrm{~g}$ concentrate mixture $+50 \mathrm{~g}$ berseem clover $+450 \mathrm{~g}$ spent rice straw. 
Table 4. Effect of replacing berseem clover with spent rice straw on blood serum parameters $(n=9)$

\begin{tabular}{|c|c|c|c|c|c|}
\hline & \multicolumn{3}{|c|}{$\operatorname{Diets}^{1}$} & \multirow{2}{*}{ SEM } & \multirow{2}{*}{$\mathrm{p}$ value } \\
\hline & Control & SRS25 & SRS45 & & \\
\hline Total proteins $(\mathrm{g} / \mathrm{dL})$ & 6.31 & 5.98 & 5.98 & 0.114 & 0.0853 \\
\hline Albumin $(\mathrm{g} / \mathrm{dL})$ & $3.04^{\mathrm{ab}}$ & $3.15^{\mathrm{a}}$ & $2.93^{\mathrm{b}}$ & 0.079 & 0.0812 \\
\hline Globulin (g/dL) & $3.27^{\mathrm{a}}$ & $2.83^{\mathrm{b}}$ & $3.05^{\mathrm{ab}}$ & 0.114 & 0.0148 \\
\hline Urea $(\mathrm{mg} / \mathrm{dL})$ & 43.5 & 42.8 & 41.7 & 0.31 & 0.1172 \\
\hline Creatinine (mg/dL) & $1.05^{\mathrm{a}}$ & $0.97^{\mathrm{b}}$ & $1.00^{\mathrm{b}}$ & 0.043 & 0.0150 \\
\hline Serum aspartate aminotransferase (units/dL) & 23.4 & 23.7 & 24.1 & 2.99 & 0.6697 \\
\hline Alanin aminotransferase (units/dL) & 14.9 & 14. 8 & 14.2 & 3.11 & 0.5925 \\
\hline Glucose $(\mathrm{mg} / \mathrm{dL})$ & $67.4^{\mathrm{a}}$ & $63.5^{\mathrm{b}}$ & $61.8^{\mathrm{b}}$ & 3.21 & 0.0002 \\
\hline Triglycerides (mg/dL) & 173.6 & 169.1 & 167.6 & 5.77 & 0.1149 \\
\hline Cholesterol (mg/dL) & $119.5^{\mathrm{a}}$ & $116.2^{\mathrm{b}}$ & $115.8^{\mathrm{b}}$ & 3.33 & 0.0016 \\
\hline
\end{tabular}

Means with different superscripts in the same row differ $(\mathrm{p}<0.05)$.

${ }^{1}$ Diets contained per kg: Control $=500 \mathrm{~g}$ concentrate mixture $+500 \mathrm{~g}$ berseem clover, $\mathrm{SRS} 25=500 \mathrm{~g}$ concentrate mixture $+250 \mathrm{~g}$ berseem clover $+250 \mathrm{~g}$ spent rice straw; $\mathrm{SRS} 45=500 \mathrm{~g}$ concentrate mixture $+50 \mathrm{~g}$ berseem clover $+450 \mathrm{~g}$ spent rice straw.

(RUP) in the SRS rations (Tomlinson et al., 1997).

The increased content of ash in rations as SRS levels increased may have been responsible for the decreased digestibility of $\mathrm{OM}$ due to the protection of fibrous materials from the attack by rumen microbes. The decreased CP digestibility in rations as SRS levels increased may be because BC contains higher levels of soluble CP compared with SRS (Table 1; Owens and Bergen, 1983), and/or because some of the $\mathrm{N}$ in SRS originates from mycelium and the mushroom fruit body. Mycelium $\mathrm{N}$ may be partly complexed with chitin that would not be easily digested (Fazaeli and Talebian Masoodi, 2006). Increased ADF and NDF digestibilities for the SRS25 and SRS45 rations may be a result of enzymatic hydrolysis by the fungus $P$. ostreatus, which reduced indigestible cell wall components and increased straw DM digestibility (Singh et al., 1990).

\section{Rumen kinetics}

Total VFAs concentration in the rumen depends on factors such as digestibility, rate of absorption, rumen $\mathrm{pH}$, rate of digesta passage from rumen to other parts of the digestive tract as well as the microbial population in the rumen and their activities (Flatt et al., 1956). A higher $\mathrm{pH}$ is favorable for bacterial adherence, an important prerequisite for fiber digestion (Palmonari et al., 2010). Compounds containing calcium used for fungal growth may be possibly responsible for the increased $\mathrm{pH}$ values with rations containing SRS.

Rumen levels of TN and NPN depends on many factors. The most important is the dietary $\mathrm{CP}$ solubility. Berseem clover contains highly soluble protein compared with the

Table 5. Effect of replacing berseem clover with spent rice straw on milk yield, milk composition and feed efficiency $(n=9)$

\begin{tabular}{|c|c|c|c|c|c|}
\hline & \multicolumn{3}{|c|}{ Diets $^{1}$} & \multirow{2}{*}{ SEM } & \multirow{2}{*}{$\mathrm{p}$ value } \\
\hline & Control & SRS25 & SRS45 & & \\
\hline Milk yield $(\mathrm{g} / \mathrm{d})$ & $966.1^{\mathrm{a}}$ & $953.7^{\mathrm{a}}$ & $802.4^{\mathrm{b}}$ & 14.22 & 0.0330 \\
\hline Fat corrected milk at $4 \%(\mathrm{~g} / \mathrm{d})$ & $880.4^{\mathrm{ab}}$ & $924.5^{\mathrm{a}}$ & $798.3^{\mathrm{b}}$ & 16.54 & 0.1402 \\
\hline Energy corrected milk (g/d) & $898.9^{\mathrm{a}}$ & $920.1^{\mathrm{a}}$ & $665.4^{\mathrm{b}}$ & 18.44 & 0.0290 \\
\hline \multicolumn{6}{|l|}{ Milk composition (g/kg) } \\
\hline Fat & $34.2^{\mathrm{b}}$ & $38.0^{\mathrm{ab}}$ & $40.1^{\mathrm{a}}$ & 2.99 & 0.0373 \\
\hline Total solids, & 125 & 128 & 129 & 4.4 & 0.3816 \\
\hline Solids not fat & 90.4 & 90.3 & 89.3 & 4.51 & 0.7055 \\
\hline Total proteins & 36.4 & 40.2 & 38.1 & 2.72 & 0.0392 \\
\hline Lactose & 47.5 & 43.6 & 42.7 & 4.51 & 0.5601 \\
\hline Ash & 6.6 & 6.9 & 8.5 & 0.27 & 0.5992 \\
\hline $\mathrm{pH}$ value & 6.90 & 6.95 & 6.86 & 0.069 & 0.8181 \\
\hline \multicolumn{6}{|l|}{ Feed efficiency } \\
\hline Fat corrected milk/DM intake & 1.25 & 1.33 & 1.17 & 1.836 & 0.1132 \\
\hline Energy corrected milk/DM intake & $1.28^{\mathrm{a}}$ & $1.33^{\mathrm{a}}$ & $0.97^{\mathrm{b}}$ & 0.102 & 0.0885 \\
\hline
\end{tabular}

Means with different superscripts in the same row differ $(\mathrm{p}<0.05)$.

${ }^{1}$ Diets contained per kg: Control $=500 \mathrm{~g}$ concentrate mixture $+500 \mathrm{~g}$ berseem clover, $\mathrm{SRS} 25=500 \mathrm{~g}$ concentrate mixture $+250 \mathrm{~g}$ berseem clover $+250 \mathrm{~g}$ spent rice straw; SRS $45=500 \mathrm{~g}$ concentrate mixture $+50 \mathrm{~g}$ berseem clover $+450 \mathrm{~g}$ spent rice straw. 
Table 6. Effect of replacing berseem clover with spent rice straw on milk fatty acids profile (mg/FA) of lactating goats $n=9$

\begin{tabular}{|c|c|c|c|c|c|}
\hline & \multicolumn{3}{|c|}{ Diets $^{1}$} & \multirow{2}{*}{ SEM } & \multirow{2}{*}{$\mathrm{p}$ value } \\
\hline & Control & SRS25 & SRS45 & & \\
\hline$\overline{C_{4: 0}}$ & 4.62 & 4.02 & 6.39 & 0.314 & 0.0410 \\
\hline$C_{6: 0}$ & 1.56 & 1.44 & 1.31 & 0.140 & 0.1475 \\
\hline $\mathrm{C}_{8: 0}$ & 2.37 & 2.53 & 2.34 & 0.145 & 0.1688 \\
\hline $\mathrm{C}_{10: 0}$ & 10.04 & 9.09 & 8.92 & 0.291 & 0.5148 \\
\hline $\mathrm{C}_{11: 0}$ & 0.10 & 0.04 & ND & 0.051 & 0.4025 \\
\hline $\mathrm{C}_{12: 0}$ & 4.30 & 3.69 & 3.73 & 0.212 & 0.8929 \\
\hline $\mathrm{C}_{13: 0}$ & ND & 0.18 & 0.03 & 0.061 & 0.2150 \\
\hline $\mathrm{C}_{14: 0}$ & 9.75 & 8.43 & 8.78 & 0.303 & 0.9575 \\
\hline $\mathrm{C}_{14.1}$ & $0.19^{\mathrm{b}}$ & $0.37^{\mathrm{a}}$ & $0.08^{\mathrm{b}}$ & 0.061 & 0.0477 \\
\hline $\mathrm{C}_{15: 0}$ & 1.46 & 0.62 & 0.52 & 0.132 & 0.2772 \\
\hline$C_{16: 0}$ & $35.65^{\mathrm{a}}$ & $25.46^{\mathrm{b}}$ & $26.37^{\mathrm{b}}$ & 0.398 & 0.1190 \\
\hline $\mathrm{C}_{16.1}$ & 1.04 & 0.95 & 0.66 & 0.104 & 0.3523 \\
\hline $\mathrm{C}_{17: 0}$ & 0.38 & 0.60 & 0.75 & 0.102 & 0.5861 \\
\hline $\mathrm{C}_{18: 0}$ & $11.26^{\mathrm{b}}$ & $14.42^{\mathrm{a}}$ & $16.15^{\mathrm{a}}$ & 0.307 & 0.2143 \\
\hline$C_{18: \ln 9 t}$ & 11.72 & 19.22 & 13.84 & 0.582 & 0.8530 \\
\hline $\mathrm{C}_{18: \ln 9 \mathrm{c}}$ & 0.49 & 2.01 & 0.72 & 0.204 & 0.4141 \\
\hline $\mathrm{C}_{18: 2}$ & ND & 0.15 & ND & 0.257 & 0.4934 \\
\hline $\mathrm{C}_{18: 2 . \mathrm{n} 6 \mathrm{t}}$ & 1.37 & 0.50 & 1.88 & 0.211 & 0.0308 \\
\hline $\mathrm{C}_{18: 2 \mathrm{n} 6 \mathrm{c}}$ & $0.68^{\mathrm{b}}$ & $2.24^{\mathrm{a}}$ & $2.73^{\mathrm{a}}$ & 0.245 & 0.1369 \\
\hline $\mathrm{C}_{20: 0}$ & 0.35 & 1.96 & 2.34 & 0.090 & 0.1402 \\
\hline $\mathrm{C}_{18: 3 \mathrm{n} 6}$ & $0.70^{\mathrm{a}}$ & $0.12^{\mathrm{b}}$ & $0.21^{\mathrm{b}}$ & 0.139 & 0.2251 \\
\hline $\mathrm{C}_{20: 1}$ & 1.55 & 0.39 & 0.63 & 0.211 & 0.4991 \\
\hline $\mathrm{C}_{20: 2}$ & 0.12 & 0.11 & 1.30 & 0.058 & 0.3179 \\
\hline $\mathrm{C}_{18: 3 \mathrm{n} 3}$ & 0.30 & 0.12 & 0.24 & 0.086 & 0.2799 \\
\hline $\mathrm{C}_{21: 0}$ & ND & 0.30 & 0.07 & 0.197 & 0.5000 \\
\hline SFA & $81.84^{\mathrm{a}}$ & $72.78^{\mathrm{b}}$ & $77.7^{\mathrm{ab}}$ & 0.555 & 0.0461 \\
\hline USFA & $18.16^{\mathrm{b}}$ & $27.22^{\mathrm{a}}$ & $22.39^{\mathrm{ab}}$ & 0.355 & 0.0752 \\
\hline MUFA & 14.99 & 22.94 & 15.93 & 0.571 & 0.7450 \\
\hline PUFA & $3.17^{\mathrm{b}}$ & $4.28^{\mathrm{ab}}$ & $6.37^{\mathrm{a}}$ & 0.291 & 0.0896 \\
\hline SCFA & 4.62 & 4.02 & 6.39 & 0.249 & 0.7410 \\
\hline MCFA & $18.37^{\mathrm{a}}$ & $16.79^{\mathrm{b}}$ & $16.30^{\mathrm{b}}$ & 0.399 & 0.4424 \\
\hline LCFA & 77.01 & 79.19 & 77.31 & 0.558 & 0.7065 \\
\hline CLA & $2.05^{\mathrm{b}}$ & $2.89^{b}$ & $4.61^{\mathrm{a}}$ & 0.252 & 0.0445 \\
\hline
\end{tabular}

Means with different superscripts in the same row differ $(\mathrm{p}<0.05)$. SFA $=$ Saturated fatty acids; USFA $=$ Unsaturated fatty acids; MUFA $=$ Monounsaturated fatty acids; PUFA $=$ Polyunsaturated fatty acids; SCFA $=$ Short-chain fatty acids (C4-C5); MCFA = Medium-chain fatty acids (C6-C12); LCFA = Long-chain fatty acids (C13-C21); USFA = Unsaturated fatty acids; CLA $=$ Conjugated linoleic acid; ND $=$ Not detected.

${ }^{1}$ Diets contained per kg: Control $=500 \mathrm{~g}$ concentrate mixture $+500 \mathrm{~g}$ berseem clover, SRS25 $=500 \mathrm{~g}$ concentrate mixture $+250 \mathrm{~g}$ berseem clover $+250 \mathrm{~g}$ spent rice straw; SRS45 $=500 \mathrm{~g}$ concentrate mixture $+50 \mathrm{~g}$ berseem clover $+450 \mathrm{~g}$ spent rice straw.

SRS, which likely led to lower ruminal TN, and NPN with the SRS rations. The high solubility of $\mathrm{BC}$ crude protein likely released more ammonia and amino acids in the rumen. Ruminal true protein- $\mathrm{N}$ can reflect the high content of true protein- $\mathrm{N}$ in the SRS, and the low degradability of this protein in the rumen compared with the highly degradable protein of $\mathrm{BC}$ in the rumen. Ammonia-N levels are consistent with ruminal TN and NPN levels. Higher ammonia $\mathrm{N}$ concentrations in the SRS groups was, associated with higher ruminal TVFA, which are indicators of higher fermentation rate in Control.

Intermediate dietary levels of soluble and degradable $\mathrm{CP}$ in SRS25 led to more microbial CP. Microbial CP has an important impact on the quantity and quality of metabolizable protein absorbed from the small intestine. Microbial protein is highly digestible in the small intestine, and its amino acid profile is close to that which ruminants require (Oba and Allen, 2003).

\section{Blood biochemical parameters}

Serum total protein reflects the nutritional status of the animal and it has a positive correlation with dietary protein intake (Kumar et al., 1980). The slight decrease of serum albumin for SRS45 and Control rations may be due to increased CP content of the Control and SRS25 rations and to higher $\mathrm{OM}$ and $\mathrm{CP}$ digestibility. The data clearly indicated no pathological lesions in the liver, since the liver is the main site of serum albumin synthesis. It is interesting that serum urea intake followed the same trend as rumen ammonia-N, where the SRS groups had lower serum urea and rumen ammonia-N levels than Control, possibly due to the linear relationship between blood urea-N and intake of degradable CP (Wright et al., 1998). Blood urea-N reflects the proportion of dietary $\mathrm{CP}$, the ratio of dietary $\mathrm{CP}$ to ruminally fermentable $\mathrm{OM}$, and postruminal protein metabolism (Roseler et al., 1993).

Serum urea and serum creatinine concentrations did not indicate catabolism of muscles protein catabolism situation and kidney function was not adversely affected by the any ration. Generally, serum creatinine is an indicator of glomerular filtration in the kidney. The higher serum glucose with Control may be due to higher OM digestibility and higher TVFA in the rumen of Control goats. It is of interest that blood serum glucose of the SRS groups followed the same trend as their milk yield, which may confirm results of Clark et al. (1977) who claimed a positive relationship between blood serum glucose and milk yield. That the SRS rations caused a decrease in serum triglyceride cholesterol concentration compared with Control may be due to the presence of mycelium and mushroom byproducts in the SRS, which may be responsible for the lower blood serum triglycerides (Sahloul et al., 2010).

\section{Milk yield and milk components}

There is little data in the literature regarding the lactational performance of lactating ruminants fed $P$. ostreatus treated rice straw. Despite the lack of differences 
in DM among the diets, there were decreases in milk yield. These findings could reflect constituents of the $\mathrm{BC}$, which provided additional energy to the goats of Control comparing with SRS25 and SRS45. For example, there were relationships between milk yield and serum glucose. The higher milk yield with Control and SRS25 diets may be due to the higher CP content of the Control and SRS25 rations and higher $\mathrm{CP}$ digestibilities and/or higher TVFA levels in the rumen of Control and SRS25 goats.

Higher milk fat levels for SRS goats could be related to the higher digestibilities of ADF and NDF of these rations and/or could be related to more VFA produced in the rumen of goats fed the SRS rations. Increases of milk protein level with the SRS rations may be due to an increased CP content of the SRS25 ration, higher CP, DM and fiber fraction digestibilities, and higher rumen fermentation of SRS25 compared to SRS45 goats.

Fatty acids originated from plasma $(60 \%)$ or by the $d e$ novo synthesis in the mammary gland from acetate and 2-hydroxybutyrate originating from rumen fermentation involving acetyl CoA carboxylase enzymes and fatty acid synthetase (Mesquita et al., 2008). Polyunsaturated acids are not synthesized by ruminants, so that their concentration in milk depends on the amount of them absorbed from the intestines. The increase in the SRS level of the diet proportionally decreased the $\mathrm{BC}$ level and hence availability of unsaturated fatty acids to be used by the mammary gland to syntheses milk lipids (Mesquita et al., 2008).

Under this study conditions, replacing $50 \%$ of $\mathrm{BC}$ in rations of Baladi lactating goats with SRS improved the performance of lactating goats without any adverse effect on animals' health. On the contrary, increasing SRS as high as $45 \%$ of total mixed ration of goats may negatively affect the performance of these goats.

\section{IMPLICATIONS}

This study has importance because it relates to reducing environmental impacts from rice straw burning. Moreover, it has economic importance because it introduces an inexpensive livestock feed which could aid in reducing endemic feed shortages for animal production in Egypt and other rice growing countries.

\section{REFERENCES}

AOAC. 1995. Official methods of analysis. 15th ed. Association of Official Analytical Chemists, Arlington, VA, USA.

Araba, M. and N. M. Dale. 1990. Evaluation of protein solubility as an indicator of over processing of soybean meal. Poult. Sci. 69:76-83.

Boyd, J. W. 2011. The interpretation of serum biochemistry test results in domestic animals. In: Veterinary Clinical Pathology,
Merck Sharp \& Dohme Corp., a subsidiary of Merck \& Co., Inc.

Cannon, D. C., I. Olitzky, and J. A. Inkpen. 1974. Proteins. In: Clinical Chemistry Principles and Techniques, 2nd Edition (Ed. R. J. Henry, D. C. Cannon, and J. W. Winkelman). Harper \& Row, Publishers, Hagerstown, MD, pp. 411-421.

Chahal, P. S. and D. S. Chahal. 1998. Lignocellulose waste: biological conversion. In: Bioconversion of waste materials to industrial products, 2nd ed (Ed. A. M. Martin). Blackie Academic and Professional, London. pp. 376-422.

Clark, J. H., H. R. Spires, R. G. Derring, and M. R. Bennink. 1977. Milk production, nitrogen utilization and glucose synthesis in lactating cows infused postruminally with sodium caseinate and glucose. J. Nutr. 107:631-644.

Dumas, B. T. and H. G. Biggs 1972. Standards methods of clinical chemistry. vol. 7. Academic Press, New York. p. 175.

Duncan, D. B. 1955. Multiple range and multiple $F$ tests. Biometrics 11:1-42.

Fazaeli, H. and A. R. Talebian Masoodi. 2006. Spent wheat straw compost of Agaricus bisporus mushroom as ruminant feed. Asian-Aust. J. Anim. Sci. 19:845-851.

Fazaeli, H., Z. A. Jelan, A. Azizi, J. B. Liang, H. Mahmodzadeh, and A. Osman. 2002. Effects of fungal treatment on the nutritive value of wheat straw. Malaysian J. Anim. Sci. 7:61-71.

Ferret, A., J. Plaixats, G. Caja, J. Gasa, and P. Prio. 1999. Using markers to estimate apparent dry matter digestibility, faecal output and dry matter intake in dairy ewes fed Italian ryegrass hay or alfalfa hay. Small Rum. Res. 33:145-152.

Flatt, W. P., R. G. Warner, and J. K. Loosli. 1956. Absorption of VFA from reticulo rumen of young dairy calves. J. Exp.Biol. 25:135.

Henry, J. B., Todd, Sanford and Davidsohn. 1974. Clinical Diagnosis and Measurement by Laboratory Methods, $16^{\text {th }}$ ed., W. B. Saunders and Co. Philadelphia PA. p. 260.

Howanitz, P. J. and J. H. Howanitz. 1984. Carbohydrates. In: Clinical diagnosis and management by laboratory methods (Ed. J. B. Henry). W.B. Saunders, Philadelphia. USA. pp. 168-169.

ISO-IDF. 2002. Milk fat-preparation of fatty acid methyl esters. International Standard ISO 15884-IDF 182: 2002.

Khattab, H. M., H. M. Gado, A. E. Kholif, A. M. Mansour, and A. M. Kholif. 2011. The potential of feeding goats sun dried rumen contents with or without bacterial inoculums as replacement for berseem clover and the effects on milk production and animal health. Int. J. Dairy Sci. 6:267-277.

Kim, Y. I., W. M. Cho, S. K. Hong, Y. K. Oh, and W. S. Kwak. 2011. Yield, nutrient characteristics, ruminal solubility and degradability of spent mushroom (Agaricus bisporus) substrates for ruminants. Asian-Aust. J. Anim. Sci. 24:15601568.

Kumar, N. U., B. Singh, and D. N. Verma. 1980. Effect of different levels of dietary protein and energy on growth of male buffalo calves. Ind. J. Anim. Sci. 51:513.

Makkar, H. P. S., O. P. Sharma, R. K. Dawra, and S. S. Negi. 1982. Simple determination of microbial protein in rumen liquor. J. Dairy Sci. 65:2170-2173.

Mesquita, I. V. U., Roberto Germano Costa, Rita de Cássia Ramos do Egypto Queiroga; Ariosvaldo Nunes de Medeiros, and Alexandre Ricardo Pereira Schuler. 2008. Profile of milk fatty acids from moxotó goats fed with different levels of manicoba 
(Manihot GlazioviiMuel Arg.) silage. Braz. Arch. Biol. Technol. 51:1163-1169.

NRC, 2001. Nutrient Requirements of Dairy Cattle, 7th ed. National Academy Press, Washington D.C.

Oba, M. and M. S. Allen. 2003. Effects of diet fermentability on efficiency of microbial nitrogen production in lactating dairy cows. J. Dairy Sci. 86:195-207.

Oei, P. 2005. Small scale mushroom cultivation, first ed. Digigrafi, Wageningen, The Netherlands.

Owens, F. N. and W. G. Bergen. 1983. Nitrogen metabolism of ruminant animals: historical perspective, current understanding and future implications. J. Anim. Sci. 57:498-518.

Palmonari, A., D. M. Stevenson, D. R. Mertens, C. W. Cruywagen, and P. J. Weimer. 2010. pH dynamics and bacterial community composition in the rumen of lactating dairy cows. J. Dairy Sci. 93:279-287.

Rabinovich, M. L., A. V. Bolobova, and Vasil'chenko. 2004. Fungal decomposition of natural aromatic structures and xenobiotics: a review. Appl. Biochem. Microbiol. 40:1-17.

Reitman, S. and S. Frankel. 1957. A colorimetric method for the determination of serum glutamic oxalacetic and glutamic pyruvic transaminases. Am. J. Clin. Pathol. 28:56-63.

Roseler, D. K., J. D. Ferguson, C. J. Sniffen, and J. Herrema, 1993. Dietary protein degradability effects on plasma and milk urea nitrogen and milk nonprotein nitrogen in Holstein cows. J. Dairy Sci. 76:525-534.

Sahloul, T. M., H. M. Emara, F. E. El-Deeb, E. F. Aboueladab, and D. H. El-Bushuty. 2010. Cultivation of mushroom using the environment residues at home and maximizing the utilization of nutrition and health. J. Agric. Biotech. 1:157-166.

Singh, K., S. N. Rai, Rakatan, and Y. W. Han. 1990. Biochemical profiles of solid state fermented wheat straw with Coprinus fimetarius. Ind. J. Dairy Sci. 60:984-990.

SAS Institute Inc. 2001. SAS/STAT user's guide. SAS Institute Inc., Cary, North Carolina.
Scheletter, G. and E. Nussel. 1975. Quantitative enzymatic Colorimetric determination of triglycerides in serum or plasma. Arbeitsmed Sozialmed Pracentimed 10: 25.

Sjaunja, L. O., L. Baevre, L. Junkkarinen, J. Pedersen, and J. Setala 1991. A nordic proposal for an energy corrected milk (ECM) formula, in EAAP publication no. 50: performance recording of animals: state of the art, proceedings of the 27th biennial session of the International Committee for Animal Recording (ICAR), Paris 2-6 July (ed. JAM van Arendonk). Wageningen Academic, The Netherlands.

Spierto, F. W., M. L. McNeil, and C. A. Burtis. 1979. The effect of temperature and wavelength on the measurement of creatinine with the jaffe procedure. Clin. Biochem. 12:18-21.

Steele, P., A. El-Hissewy, and A. E. Badawi 2009. Technical Manual: Agro-Industrial Use of Rice Straw. FAO. Project TCP/EGY/3102 straw with sewage sludge and compost effects on the soil-plant system. Chemosphere 75:781-787.

Stein, E. A. 1986. Textbook of chemical chemistry (Ed. N. W. Tiez). W. B. Saunder, Philadelphia. pp. 879-886.

Tawffek, J. A. 2011. Comparison study between using two kinds of Pleurotus ostreatus mushroom for improving barley straw. J. Food Industries Nutr. Sci. 1:71-74.

Tomlinson, D. L., R. E. James, G. L. Bethard, and M. L. McGilliard. 1997. Influence of undegradability of protein in the diet on intake, daily gain, feed efficiency, and body composition of Holstein heifers. J. Dairy Sci. 80:943-948.

Van Soest, P. J., J. B. Robertson, and B. A. Lewis 1991. Methods for dietary fiber, neutral detergent fiber, and non-starch polysaccharides in relation to animal nutrition. J. Dairy Sci. 74:3583-3597.

Warner, A. C. J. 1964. Production of volatile fatty acids in the rumen. Methods of Measurements. Nutr. Abst. Rev. 34:339352.

Wright, T. C., S. Moscardini, P. H. Luimes, P. Susmel, and B. W. McBride. 1998. Effects of rumen-undegradable protein and feed intake on nitrogen balance and milk protein production in dairy cows. J. Dairy Sci. 81:784-793. 